\title{
Erratum: Looking for axion dark matter in dwarf spheroidal galaxies [Phys. Rev. D 98, 083024 (2018)]
}

\author{
Andrea Caputo, Carlos Peña Garay, and Samuel J. Witte
}

(Received 14 March 2019; published 29 April 2019)

DOI: 10.1103/PhysRevD.99.089901

Two mistakes were made in the preparation of this manuscript. The first was a numerical bug that entered exclusively in the creation of Fig. 1. Here, a numerical conversion factor was improperly squared. This has been fixed, and a revised Fig. 1 is included below-note that here we have taken $f_{a} \sim 10^{-6}$ rather than $f_{a} \sim 1$, although we emphasize that realistic values of this suppression factor for large scale galactic environments are typically many orders of magnitude smaller (e.g., $f_{a}$ is expected to be $\sim 10^{-13}$ for the Galactic center). The conclusions of this plot are not significantly unaffected. The second error, however, is more significant. We had adopted an effective area from a paper which is only valid should the radio telescope be treated as an interferometer (which would required adopting a smaller field of view that is dependent upon the configuration of the telescopes). We correct for this by assuming SKA has 5600 telescopes operating independently. The reduces the sensitivity presented in Fig. 3 significantly. The modified version of Fig. 3 is shown below.

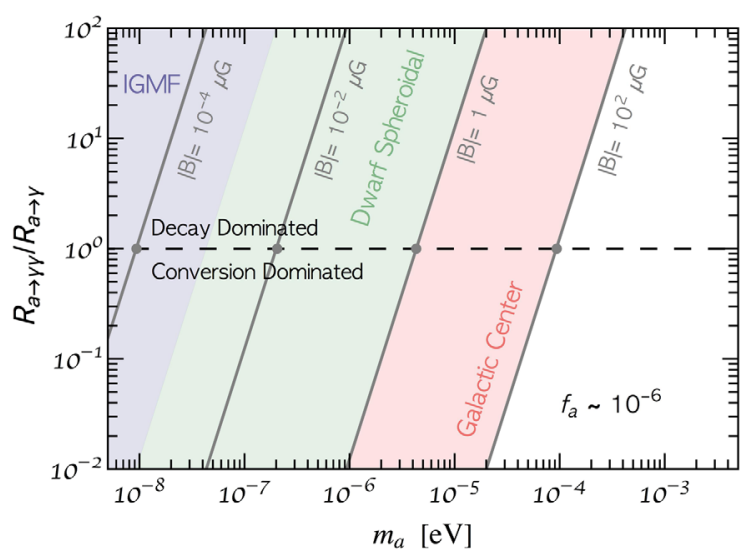

FIG. 1. Replacement of Fig. 1.

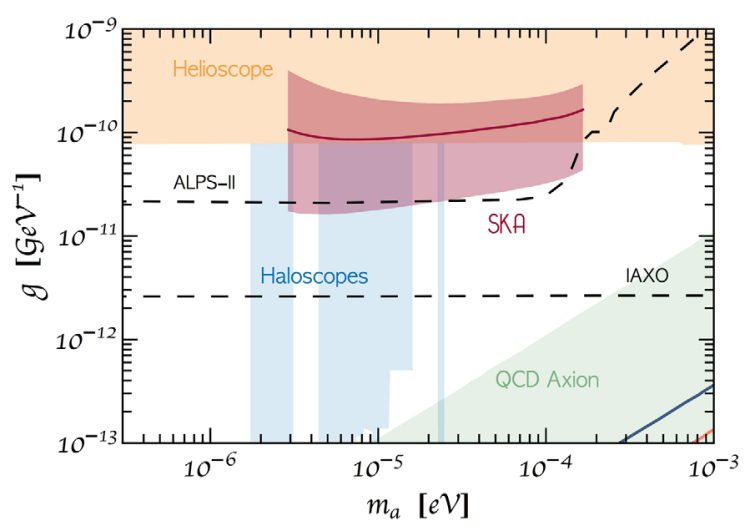

FIG. 2. Replacement of Fig. 3. 